\title{
Direct Observation of Hafnia Structural Phase Transformations
}

\author{
Bethany M. Hudak ${ }^{1}$, Sean W. Depner ${ }^{2}$, Gregory R. Waetzig ${ }^{3,4}$, Sarbajit Banerjee ${ }^{3,4}$, Beth S. Guiton ${ }^{1,5}$ \\ 1. Department of Chemistry, University of Kentucky, Lexington, Kentucky, USA \\ 2. Department of Chemistry, University at Buffalo, The State University of New York, Buffalo, NY, USA \\ 3. Department of Chemistry, Texas A\&M University, College Station, TX, USA \\ 4. Department of Materials Science and Engineering, Texas A\&M University, College Station, TX, USA \\ 5. Materials Science and Technology Division, Oak Ridge National Lab, Oak Ridge, TN, USA
}

The continued miniaturization of electronic device components such as metal oxide field effect transistors has pushed the semiconductor industry to replace silicon dioxide with high- $\kappa$ dielectrics as the gate material. Hafnia is a wide band-gap and high dielectric constant material that is thermally stable on Si substrates, making it an attractive candidate to replace $\mathrm{SiO}_{2}$ as the gate dielectric material in such devices [1]. The tetragonal phase of hafnia, occurring at $1720^{\circ} \mathrm{C}$, is desired for device application due to its larger band gap and high permittivity in comparison to the monoclinic phase [1,2]. However, due to the displacive nature of the structural phase transformation, quenching the tetragonal phase to room temperature leads to a reversion back to the monoclinic structure [2]. It has been shown that the tetragonal phase of $\mathrm{ZrO}_{2}$, an isomorph of $\mathrm{HfO}_{2}$, can be stabilized with respect to the monoclinic phase at room temperature below a critical size of about $15-30 \mathrm{~nm}$. It is estimated that the critical size for stabilization of tetragonal $\mathrm{HfO}_{2}$ is much smaller, at about 4-10 nm, thus making it more difficult to stabilize [3]. This is illustrated schematically in Figure 1a. Since the tetragonal phase of hafnia is more important for technological applications than the monoclinic phase, it is of utmost importance to find a way to stabilize the tetragonal phase at low temperature [1]. Here we observe directly the monoclinic $\rightarrow$ tetragonal structural phase transformation of an individual hafnia nanorod with atomic resolution, using in situ scanning transmission electron microscopy (STEM).

High aspect ratio, monoclinic hafnia nanorods were grown via a nonhydrolytic sol—gel synthesis. The monoclinic nanorods contain multiple twin boundaries on the (100) planes [3]. These twin planes are believed to develop to accommodate strain during the tetragonal to monoclinic phase transformation upon cooling during synthesis. Shown in Figure 1b, when heated in situ in the STEM at $600{ }^{\circ} \mathrm{C}$ - over $1000{ }^{\circ} \mathrm{C}$ below the bulk transition temperature - we can observe an atomic-scale structural phase transformation from the monoclinic phase to the tetragonal phase, and upon slow cooling we observe reduction of the nanorods to hafnium metal. The phase transformation nucleates at a twin boundary and propagates one lattice plane at a time via a transformation dislocation. The high-energy twin planes as well as surface energy effects arising from size-confinement combine to depress the transition temperature by more than $1000{ }^{\circ} \mathrm{C}$ and prevent the tetragonal to monoclinic transformation. Using these in situ techniques, we are able to study the transformation mechanism in real time, providing crucial information necessary to stabilizing tetragonal $\mathrm{HfO}_{2}$ nanocrystals near room temperature. [4] 


\section{References:}

[1] LG Wang et al, Appl. Phys. Lett. 104 (2014) p. 201903.

[2] J Tang et al, Adv. Funct. Mater. 15 (2005) p. 1595.

[3] SW Depner et al, ACS Nano 8 (2014) p. 4678.

[4] The authors acknowledge funding from NASA Kentucky under NASA Award No: NNX10AL96H, and by the National Science Foundation under DMR 1504702 and DMR 145515. The research was supported in part by the Office of Basic Energy Sciences, Materials Sciences and Engineering Division, U.S. Department of Energy, and in part by ORNL's Center for Nanophase Materials Sciences.

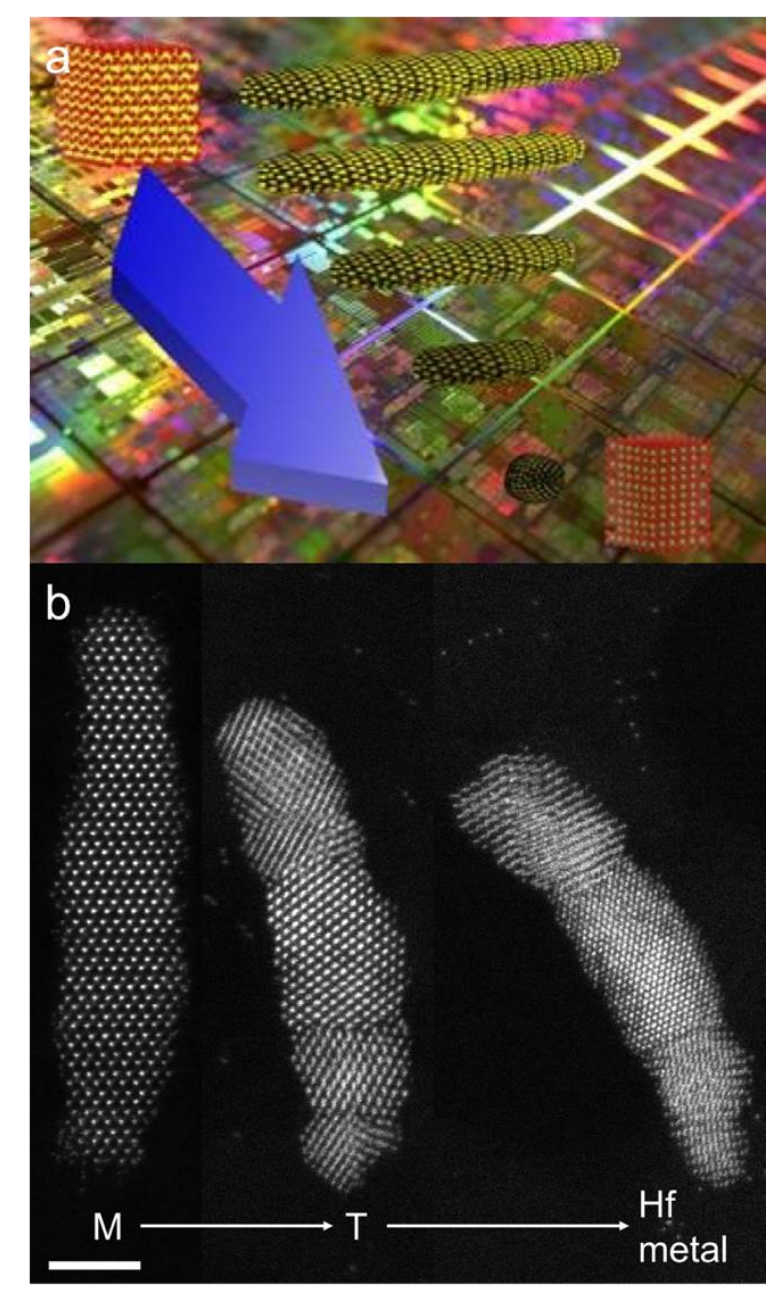

Figure 1. (a) Cartoon schematic demonstrating size confinement of hafnia nanocrystals stabilizing the tetragonal phase. (b) STEM images of a monoclinic nanorod undergoing a structural phase transformation to tetragonal $\mathrm{HfO}_{2}$ upon heating and then hafnium metal when cooled in situ. Scale bar $=5 \mathrm{~nm}$. 